\section{Métodos Substitutivos no Ensino Prático de Fisiologia}

Recibido: 1 Julio 2015 - Revisado: 30 Septiembre 2015

Aceptado: 30 Octubre 2015 - Publicado: 30 Diciembre 2015

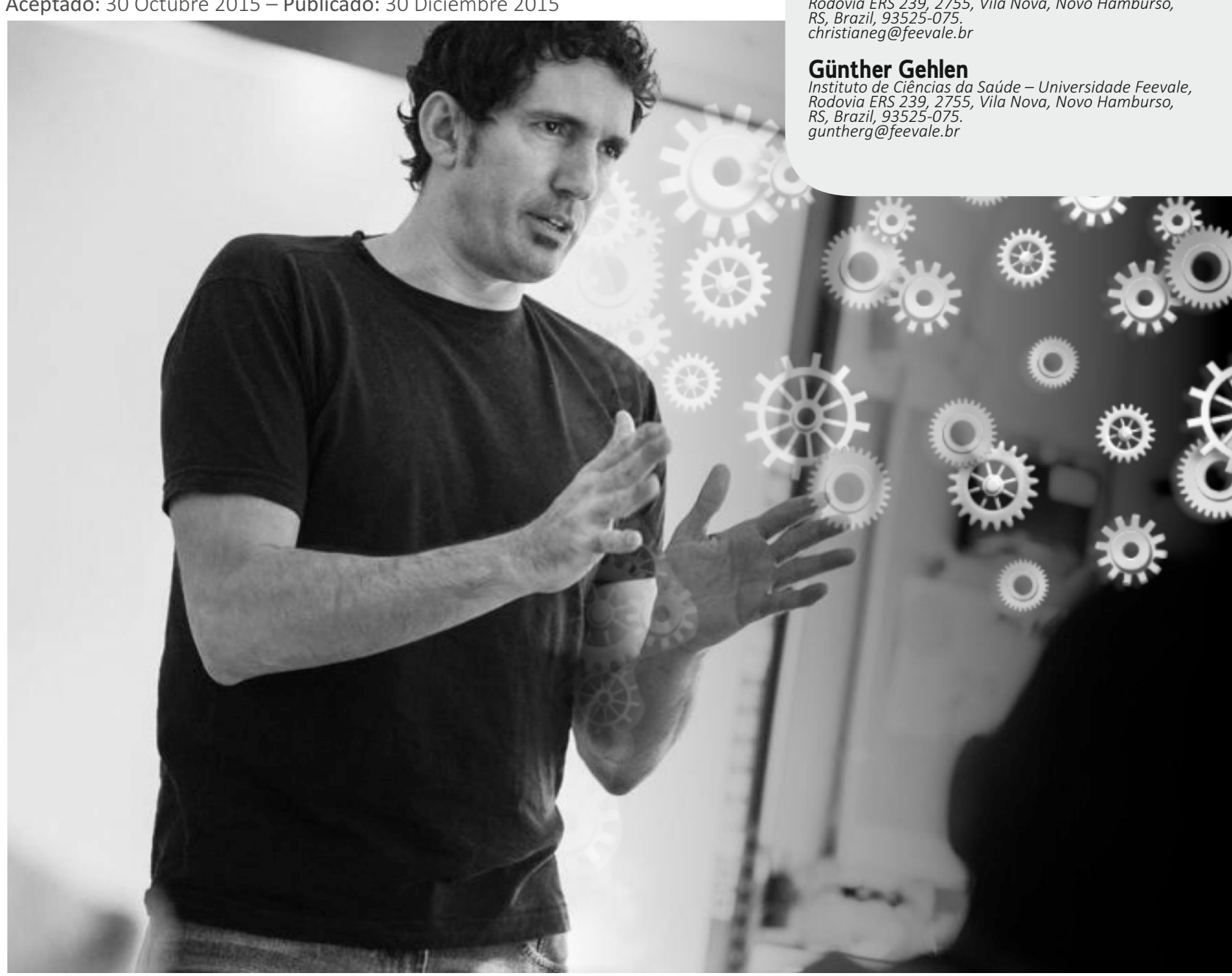

Abstract: Physiology teaching has been always associated to the use of laboratory animals. Since the current discussion about the real need of laboratory animals in research and education, and the strong local and international recommendation for animal use reduction, physiology teachers are facing the challenge of rethinking the physiology lab lessons. The aim of this work is to report the animal replacement approaches taking place at Universidade Feevale, as well as the point of view of those involved.

Resumo: O ensino de Fisiologia sempre se baseou no uso de animais de laboratório. Com a atual discussão sobre a necessidade do uso de animais em pesquisa e ensino e as recomendações tanto nacionais quanto internacionais para sua redução, os professores dessa disciplina enfrentam o desafio de reformular seu ensino prático. Esse trabalho visa relatar o processo de substituição em andamento na Universidade Feevale, juntamente com a visão daqueles que estão envolvidos. Palavras-chave: ensino prático, fisiologia, uso de animais no ensino 


\section{INTRODUÇÃO}

Ao final da década de 50 foi publicado um manual sugerindo uma série de práticas para humanização do uso de animais em pesquisa. Inicialmente a proposta atraiu pouca atenção, mas, lentamente, foi se tornado um processo em larga escala, despertando a consciência no uso de animais em atividades de pesquisa e ensino. Uma regra simples, constituída de apenas três palavras, passou a ser o centro da discussão no uso de animais: Reduction, Refinement, Replacement (Reduzir, Refinar e Substituir) comumente referida como regra dos três erres (3Rs). Os currículos de ensino na área da saúde tradicionalmente envolvem o uso de animais, uma prática que persiste até hoje nos mais diversos países, incluindo aqueles do continente Europeu, que lidera as práticas de substituição . Em 1987 um aluno norteamericano processou sua escola por obrigar os alunos a participarem de aulas de dissecação animal para o aprendizado de anatomia. A partir dessa iniciativa, diversos alunos entraram com petições semelhantes, desencadeando um processo de reflexão por parte de diversas entidades de ensino. Universidades norteamericanas como Harvard, Columbia, Mayo e Yale, já contam com laboratórios "não-vivos" para acomodar a demanda pelo ensino com sacrifício reduzido de animais .

Dentre as disciplinas dos cursos da saúde, a fisiologia sempre se baseou no uso de animais para ensino prático, visto sua complexidade e a necessidade da visualização da interação entre diferentes órgãos e sistemas para seu melhor entendimento. Com a popularização da regra dos $3 R$ s um grande desafio foi lançado aos professores dessa disciplina. Embora o uso de animais para ensino não tenha sido proibido, as discussões atuais no tema sugerem fortemente que alternativas sejam criadas, sendo apoiado pela legislação vigente. A virtualização das práticas demonstrativas passou a ser a alternativa mais viável . Uma série de jogos, aplicativos e vídeo-aulas foi desenvolvido e se encontra disponível tanto de forma gratuita como para compra. Exemplos importantes nesse contexto são os softwares de ensino Interactive Physiology da Pearson Education, os sistemas PowerLab/Labtutor da ADInstruments, bonecos de simulação da SIEMENS, dentre outros.

Dentro dessa temática, foi proposto aos professores de Fisiologia da Universidade Feevale o desafio de reformular as estratégias de ensino, principalmente o ensino prático. Duas estratégias foram divisadas para atender a essa demanda. A primeira consiste em utilizar o sistema PowerLab da ADInstruments. A segunda, se baseia no uso de bonecos de simulação.

\section{METODOLOGIA SUBSTITUTIVAS}

\subsection{Prática 1 - Contração Muscular}

Tradicionalmente, a aula prática de contração muscular é realizada a partir do isolamento do músculo gastrocnêmico de uma rã previamente anestesiada. Diferentes estímulos são aplicados sobre o músculo isolado e as respostas são observadas .

Para ilustrar na prática os aspectos mais relevantes da função neuromuscular, realizamos uma prática baseada no sistema PowerLab da ADInstruments. Nessa prática um aluno voluntário sofre estímulos elétricos no nervo ulnar/radial e a contração do adutor do polegar é registrada por um sensor de força, através de um software específico. Com essa prática é possível visualizar os efeitos de diferentes intensidades de estímulo, redução do intervalo e somação de um número crescente de estímulos sobre a força de contração muscular.

\subsection{Prática 2-Reflexos Medulares}

A prática clássica de reflexos medulares consiste em utilizar uma rã anestesiada. O objetivo da prática é demonstrar que um reflexo medular depende apenas da medula espinhal e ocorre independentemente de se ter um córtex cerebral intacto. Inicialmente um estímulo doloroso é aplicado na pata do animal e a resposta de retirada é visualizada. Imediatamente 
após, a ligação entre encéfalo e medula é destruída e se verifica que o reflexo ainda permanece, mesmo com o animal descerebrado .

Substituímos essa prática por uma versão de maior aplicabilidade clínica e envolvimento direto dos alunos. Utilizando o sistema PowerLab da ADIntruments, registramos a evocação de um reflexo patelar com o aluno atento ao movimento. Logo após, o procedimento de evocação é repetido com o aluno executando uma manobra de força com os braços, em que a sua atenção é afastada do membro que está sendo estimulado, conhecido como manobra de Jendrassik . Executar a manobra tem o mesmo efeito que descerebrar a rã: retira a inibição cortical do movimento, mostrando que ele dependente apenas da medula espinhal para ocorrer, mas pode sofrer inibição cortical .

Essa abordagem tem como desvantagem o fato de que a manobra nem sempre funciona como esperado. As distrações de se ter vários alunos em uma sala podem interferir com a abordagem. As vantagens da prática consistem em se testar a fisiologia diretamente em humanos, aproximando essa disciplina básica da prática clínica futura do profissional.

\subsection{Prática 3-Espirometria}

A prática espirométrica é tradicionalmente realizada em humanos, com uso de espirômetros analógicos, como o espirômetro com pistão . A aquisição do sistema PoweLab da ADIntruments permitiu a digitalização da prática, ampliando o número de variáveis que podem ser registradas e calculadas.

O uso de espirômetros analógicos permite a medida dos volumes pulmonares: volume corrente, volume de reserva expiratório, volume de reserva inspiratório e, a partir desses valores, o cálculo das capacidades inspiratória, expiratória e vital. A visualização da função pulmonar ficar dificultada, pois a medida dos fluxos não está presente nesse tipo de avaliação .
Com o uso de sistemas digitais, todos os valores anteriores podem ser medidos além de se estimar o volume residual, permitindo o cálculo da capacidade pulmonar total e a Capacidade Vital Forçada (CVF). Como os volumes e fluxos são registrados em um software, é possível realizar a análise posterior, obtendo-se medidas da função pulmonar como o Volume Expirado Forçadamente em 1 segundo (VEF1) e o índice de Tiffeneau (VEF1/CVF).

\subsection{Prática 4-Eletrocardiograma}

O sistema PowerLab também permite o registro de um eletrocardiograma simples, com as três derivações clássica DI, DII e DIII. Anteriormente, essa prática era realizada de forma demonstrativa o que não permitia a todos o manuseio do aparelho para um melhor aprendizado. Com a prática interativa, observamos um maior interesse em participar da atividade, tanto no momento da aula, como na pesquisa sobre o assunto em horário extraclasse. Trabalhando em grupos, os alunos podem comparar os resultados e discutir as diferenças de acordo com o gênero, peso, altura, condição física e de saúde e etnia.

Na prática de eletrocardiograma (ECG), os alunos identificam as ondas P e T e complexo QRS, a frequência cardíaca, os principais segmentos e intervalos, analisando os resultados com dados de referência para verificar se os seus dados estão normais . Para qualquer alteração fora do normal é ressaltado que o aparelho é um simulador que pode sofrer interferências por vários fatores.

\subsection{Prática 5 - Efeitos do sistema autônomo sobre o sistema cardiovascular}

Algumas práticas ainda estão em fase de adequação e não foram implementadas. As práticas 5 e 6 utilizam um boneco de simulação, e dependem de turmas menores para sua implementação.

A abordagem prática dos efeitos do sistema nervoso autônomo sobre o sistema cardiovascular é 
tradicionalmente realizada com coração isolado e mesentério de rã. É uma prática trabalhosa e de visualização difícil .

Com bonecos de simulação existe a possibilidade de simular a injeção de adrenalina, acetilcolina e seus fármacos agonistas e antagonistas para verificar alterações de parâmetros como frequência cardíaca, pressão sanguínea arterial e ECG. O boneco passa a ser um paciente. Frente a uma alteração de pressão, por exemplo, os alunos devem propor soluções. As respostas são então testadas no boneco e se verifica a melhora ou piora do quadro.

\subsection{Prática 6-Controle ácido-básico}

O uso de bonecos de simulação também permite avaliar o controle ácido-básico efetuado pelo sistema respiratório. Através de simulação de modificação do $\mathrm{pH}$ do sangue (simulando uma alcalose ou acidose metabólica) se pode observar como alterações da frequência respiratória regulam o pH do sangue.

Poderemos visualizar o efeito que alteração da frequência respiratória por doença pulmonar obstrutiva crônica (DPOC) têm sobre o pH do sangue, deixando-o mais ácido. É possível visualizar também os efeitos da hiperventilação desencadeada por alterações de humor, como ansiedade e nervosismo e a consequente alcalinização do sangue.

O mais interessante dessas abordagens é o envolvimento do aluno na resolução de problemas, vendo na pratica a causa e a solução de alterações fisiológicas.

\section{RESULTADOS E PERCEPÇÕES}

Os métodos aqui apresentados vêm sendo desenvolvidas e aplicadas nos últimos três semestres, com aplicação em maior escala a partir do semestre passado. Embora a experiência seja recente e esteja em fase de adaptação, alguns resultados já podem ser observados. As práticas selecionadas nesse trabalho abordam pontos sensíveis do aprendizado em fisiologia, em que a grande maioria dos alunos apresentam dificuldades de compreensão.

Dentro da prática de contração muscular, os conceitos de potencial de ação, sua condução e somação temporal tiveram sua compreensão facilitada pela abordagem prática. Se ainda estivéssemos utilizando métodos clássicos, esses conceitos não seriam abordados de forma prática e continuariam uma temática de difícil compreensão. A visualização do processo fisiológico na prática, torna um conceito abstrato em algo palpável, aplicável no cotidiano.

A possibilidade de quantificar os reflexos medulares em um voluntário humano, enriqueceu a compreensão da sua fisiologia e despertou o interesse da turma. A discussão em torno dessa prática tem sido construtiva com a participação maciça dos alunos.

A ampliação das medidas que a espirometria digital traz, envolve os alunos em uma prática que anteriormente era considerada tediosa. A possibilidade de saber a eficiência e funcionalidade dos seus próprios pulmões engaja o aluno na prática, tornado a experiência mais enriquecedora, o mesmo sendo aplicado à pratica de eletrocardiograma.

Até o presente momento, desenvolvemos as aulas práticas e medimos sua aplicabilidade. As melhoras no processo de aprendizagem foram medidas de forma qualitativa, pela observação do desempenho das turmas em comparação com a experiência dos docentes com turmas de semestres anteriores. Processos de mensuração quantitativa do ganho no processo de ensino com o uso das novas metodologias estão sendo planejados para aplicação em um futuro próximo.

De forma geral, a virtualização das práticas em fisiologia traz mais ganhos do que perdas. Embora o 
investimento técnico e financeiro inicial seja grande, a possibilidade de se utilizar os próprios alunos como voluntários e o número de parâmetros sendo registrados, superam os resultados que poderiam ser obtidos a partir do uso de um animal.

\section{CONCLUSÕES}

Durante muitos anos o ensino prático em fisiologia tem sido uma repetição dos métodos aprendidos pelos professores quando alunos. O movimento de redução do uso de animais em metodologias de ensino serviu como choque despertador, levando à reformulação das metodologias clássicas.

Mudar o processo vigente é extremamente desafiador. Deixar de usar técnicas clássicas, consolidadas, retira o educador da sua área de conforto. Não obstante, passado o choque inicial e atingida a energia de ativação para o desenvolvimento de novas técnicas, os resultados vêm sendo recompensadores. O uso das tecnologias digitais tem permitido explorar conceitos antes ignorados, além de aproximar o profissional da saúde em formação da sua futura atuação clínica.

Com as novas abordagens, deixamos um pouco o aspecto de ciência básica, mais comumente atribuído à fisiologia, transformando-a em uma ciência de integração básico-clínica, mais condizente com o caráter multidisciplinar e translacional dessa disciplina.

\section{REFERÊNCIAS}

(2008) "Lei Arouca- 11.794".

Abruzzi Júnior, J. (2010) "Eletrocardiograma". In: Fisiologia Prática. Edited by EDUCS. Caxias do Sul,

Badyal, D. K. \& Desai, C. (2014). Animal use in pharmacology education and research: the changing scenario. In Indian J Pharmacol, 46, 257-65.

Balls, M. (1994). Replacement of animal procedures: alternatives in research, education and testing. In Lab Anim, 28, 193-211.

Bosi, H. R. (2010) "Efeitos do neurotrasmissores do autonômicos sobre o coração e o sistema gastrointestinal da rã". In: Fisiologia Prática. Edited by EDUCS. Caxias do Sul,

Braga, G. L. (2010) "Rã Espinhal e Rã Descerebrada". In: Fisiologia Prática. Edited by EDUCS. Caxias do Sul,

Brollo, L., Bombig, M. T. N., Mazzaro, C. D. L., Francisco, Y. A., Fonseca, F. a. H., Carvalho, A. C. C., Harima, H., Hirai, A. \& Povoa, R. (2009). Associação do eletrocardiograma com diabete melito e síndrome metabólica em nipo-brasileiros. In Arquivos Brasileiros de Cardiologia, 92.

Cingolani, H. E. \& Houssay, A. B. (2004) "Fisiologia Humana de Huoussay", Artmed, 7ạ.

Costa, D. \& Jamami, M. (2001). Bases fundamentais da espirometria. In Revista Brasileira de Fisiologia, 5, 95102.

Curzer, H. J., Perry, G., Wallace, M. C. \& Perry, D. (2015). The three Rs of animal research: What they mean for the Institutional Animal Care and Use Committee and why. In Sci Eng Ethics.

Dewhurst, D. \& Ward, R. (2014). The virtual 
pharmacology lab - a repository of free educational resources to support animal-free pharmacology teaching. In Altern Lab Anim, 42, 4-8.

Dewhurst, D. G. \& Kojic, Z. Z. (2011). Replacing animal use in physiology and pharmacology teaching in selected universities in Eastern Europe--charting a way forward. In Altern Lab Anim, 39, 15-22.

Dias, A. S. (2001) "Prática de Espirometria". In: Fisiologia Prática. Edited by Editora ULBRA. Canoas,

Doke, S. K. \& Dhawale, S. C. (2015). Alternatives to animal testing: A review. In Saudi Pharm J, 23, 223-9.

Flecknell (2002) "Replacement, Reduction and Refinement" Use of animals in research: a sciencesociety controversy? Mainz, Alemanha.

Hayes, K. C. (1972). Jendrassik maneuver facilitation and fractionated patellar reflex times. In Journal of Applied Physiology, 32, 5.

Madsen, F., Mortensen, J., Hanel, B. \& Pedersen, O. F. (2014) "Lung Function Testing, Spirometry, Diffusion Capacity and Interpretation". In: Mechanics of Breathing. Edited by Springer Milan. Milão,

Mullane, M., Maloney, S. J., Chavda, S., Williams, S. \& Turner, A. N. (2015). The Effects of Concurrent Activation Potentiation on Countermovement Jump Performance. In J Strength Cond Res.

Mundt, C. S., Marroni, N. P. \& Kauer, V. S. (2001) "Contração do músculo gastrocnêmico da rã". In: Fisiologia Prática. Edited by Editora ULBRA. Canoas,

Ribeiro, M. F. M. (2001) "Controle medular e supramedular da atividade motora em rãs". In: Fisiologia Prática. Edited by Editora ULBRA. Canoas,

Rossi, I. C. C. \& Vinagre, A. P. (2001) "Efeito de Neurotransmissores do Sistema Nervoso Autônomo
Sobre o Coração e Órgãos Gastrointestinais". In: Fisiologia Prática. Edited by Editora ULBRA. Canoas,

Rowan, A. N. (1980). The concept of the three R's. An introduction. In Dev Biol Stand, 45, 175-80.

Russel, W. M. S. \& Burch, R. L. (1959) "The principles of humane experimental technique", Methuen Co. Ltd,

Sartori, G. (2010) "Protocolo de Espirometria". In: Fisiologia Prática. Edited by EDUCS. Caxias do Sul,

Weihe, W. H. (1985). Use and misuse of an imprecise concept: alternative methods in animal experiments. In Lab Anim, 19, 19-26.

Zeng, R., Yue, R. Z., Tan, C. Y., Wang, Q., Kuang, P., Tian, P. W. \& Zuo, C. (2015). New ideas for teaching electrocardiogram interpretation and improving classroom teaching content. In Adv Med Educ Pract, 6, 99-104. 